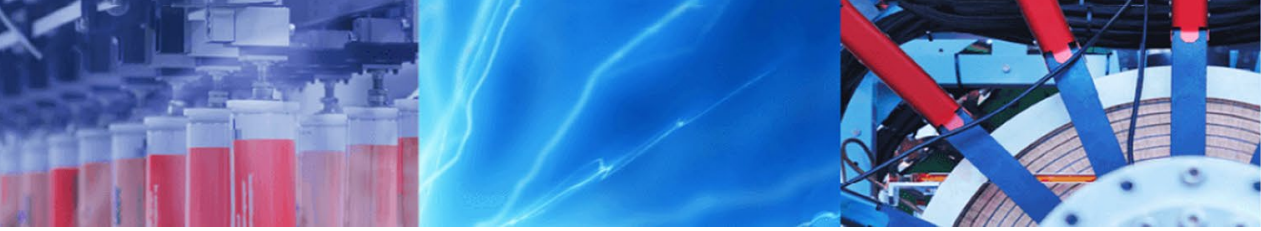

Research Article

\title{
Parametric optimization of fused deposition modeling using learning enthusiasm enabled teaching learning based algorithm
}

\author{
Jasgurpreet Singh Chohan ${ }^{1}$ (D) Nitin Mittal ${ }^{1} \cdot$ Raman Kumar $^{1}$
}

Received: 1 March 2020 / Accepted: 31 October 2020 / Published online: 10 November 2020

(c) Springer Nature Switzerland AG 2020

\begin{abstract}
This study presents an advanced Learning Enthusiasm based teaching-learning algorithm (LebTLBO) for optimization of line width compensation, extrusion speed, filling speed, and layer thickness of the Fused Deposition Modeling (FDM) process. These independent parameters have a notable effect on dimensional accuracy, warp deformation, and manufacturing time which further affects the usability of FDM parts. A comparative analysis of LebTLBO has been performed with conventional algorithms for solving a complex optimization problem. This optimization tool simulates the classroom's teaching-learning process and demands only the common control parameters i.e. number of generations and population size instead of algorithm-specific control parameters. The findings show that LebTLBO is most efficient to chalk out single parametric settings which would maximize each response. The optimum value of parameters predicted by LebTLBO is $0.15 \mathrm{~mm}$ layer thickness, extrusion speed of $21.85 \mathrm{~mm} / \mathrm{s}$, and $40 \mathrm{~mm} / \mathrm{s}$ filling speed for acrylonitrile butadiene styrene thermoplastic material. Confirmatory experiments validate the efficiency of LebTLBO as the maximum response is achieved at predicted parametric settings.
\end{abstract}

Keywords Fused deposition modeling · Optimization · Teaching learning based algorithm · Dimensional accuracy · Simulation · Learning enthusiasm

\section{Introduction}

In this ever-changing market scenario, there is an increasing demand for technology that can efficiently meet customer needs of high-quality and low-cost products. Moreover, there is a requirement of flexible manufacturing processes that can rapidly supply products with the highest aesthetic value without environmental degradation [1]. Additive manufacturing technologies have revolutionized the production processes by reducing product manufacturing time by $50 \%$. These digital manufacturing processes can fabricate custom made parts directly from a computer-generated three-dimensional model. Another breakthrough in this field is the fabrication of patientspecific implants at a comparatively lesser cost and time
[2]. As opposed to traditional manufacturing techniques, this technology fabricates a part progressively in the form of small layers stacked one upon another [3]. Due to the merits of additive manufacturing technologies, these have been implemented into numerous fields, including the automotive, aerospace, jewelry, sports electronics, consumer goods, toys, and medical industries [4].

Presently, there are number of digital fabrication techniques but Fused Deposition Modeling (FDM) is selectively used to fabricate end-use components due to material flexibility, simple operation, and portability of machine. In FDM, initially, the part is designed in suitable designing software and transformed to Standard Tessellation Language (STL) format for further processing. Afterwards, toolpaths are generated by slicing software which

Jasgurpreet Singh Chohan, jaskhera@gmail.com | 'University Centre for Research and Development, Chandigarh University, Mohali 140413, India. 
tessellates the part into small slices instead of complex structure [5]. In Fused Deposition Modeling, layer-by-layer deposition of plastic filament is automatically controlled by three stepper motors (for $\mathrm{X}, \mathrm{Y}$ and $\mathrm{Z}$ direction) based on toolpaths developed by slicing software. These toolpaths further depend upon orientation angle, density, and internal raster arrangement which can be customized before fabrication [6].

The extruder head comprises of rollers and a heated nozzle where plastic filament is supplied and deposited

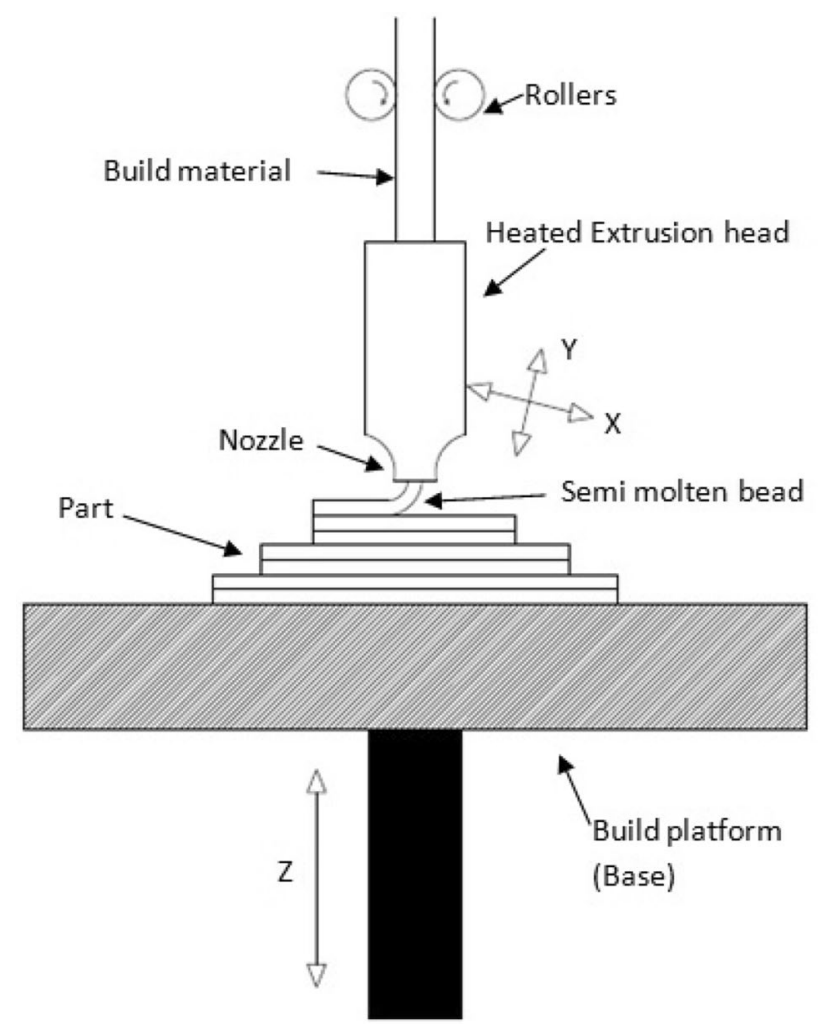

Fig. 1 Working Principle of FDM process on build platform (Fig. 1). The build material in form of thin wire which is heated at temperature minutely below the melting point so that semi-molten bead is precisely layered on platform. The extruder head accelerates both in $\mathrm{X}$ and $\mathrm{Y}$ direction whereas table moves in $\mathrm{Z}$ axis which results in three-dimensional deposition of semi-molten plastic filament. Besides part material, water-soluble support material is also extruded by another nozzle to provide strength to over-hanging parts. The part is fabricated within few hours and ready to use after removal of support structures. Nowadays, commercial FDM machines have provisions to alter different process parameters to achieve desired characteristics in final parts [7].

In FDM parts, dimensional accuracy is a major limitation as the components are fabricated step by step which induces considerable surface roughness and inaccuracy. Many attempts have been made to identify optimum parameters during pre-processing and post-processing operations which would yield maximum dimensional stability and accuracy of FDM parts [8]. Figure 2 depicts different process parameters of FDM which have been extensively studied by researchers.

Recent research has been focused on optimization of FDM process parameters as a slight change in parameters has a drastic impact on the output characteristics [9]. Since dimensional accuracy is of the highest priority in the case of direct functional parts. Most researchers have executed optimization studies to reduce irregularities appearing on upper surface which cause dimensional variability. Pandey et al. [2] used a modified version (genetic algorithm-II) intending to optimize the orientation angle to achieve minimum production time and surface roughness. Generally, surface roughness and production time are inversely proportional to each other as the reduction in one objective function would invariably increase another but algorithms were developed to resolve this problem and select the best orientation angle. The complex parts
Fig. 2 Different process parameters of FDM [7]

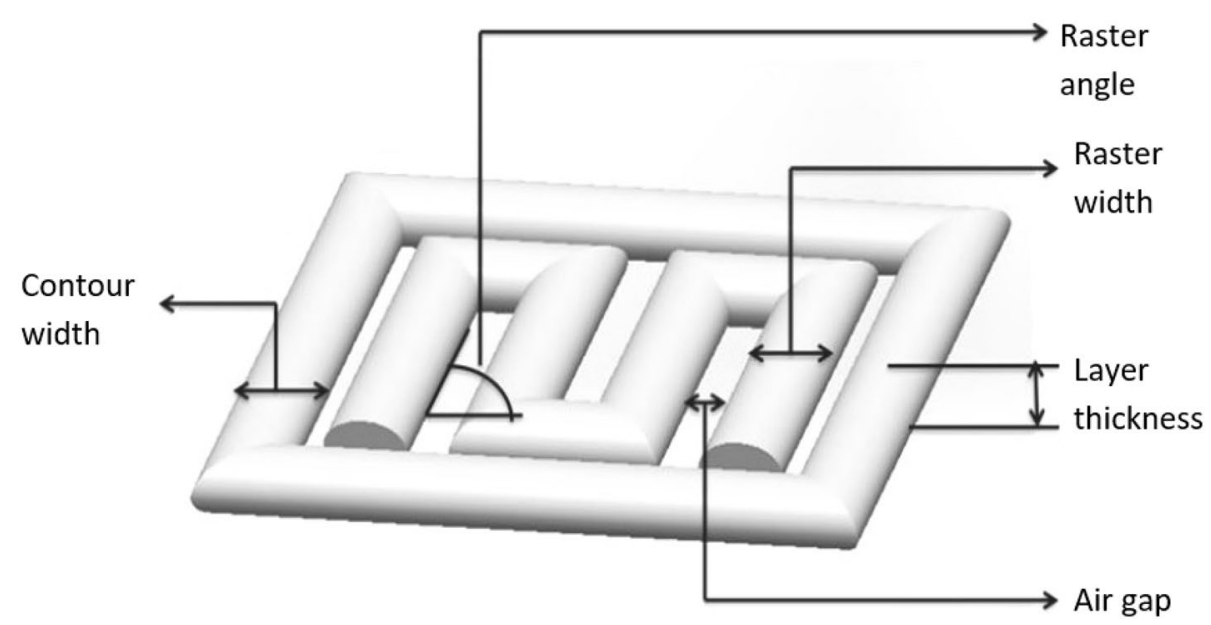


were deliberately selected so as to validate the study for practical problems and it was found that the algorithms can be used for optimization of any geometry. Ahn et al. [4] presented a methodology to estimate the surface asperities based on theoretical and actual conditions. The equations were generated to predict the surface finish of FDM parts in terms of deposition angle. The predicted data was compared with actual measurements for validation and minimum error was found at specific angles. Mahapatra and Sood [10] utilized artificial neural networks to predict the surface irregularities of FDM components while investigating five parameters i.e. layer thickness, deposition angle, air gap, raster angle, and width. Bayesian regularization has been implemented to identify optimum network architecture and the model was trained using Levenberg-Marquardt algorithm to eliminate over-fitting chances. It is highly effective method for prediction as the algorithm approaches the second-order training speed requirement of Hessian matrix. Boschetto et al. [11] formulated an equation using geometric modelling of upper surface profile as a function of layer thickness and orientation angle to estimate the average roughness of FDM parts. Initially, number of parts were manufactured at variable parameters and it was found that deposition angle has maximum impact on surface roughness. The modelled data was compared with experimental results and higher correlation was found between both conforming the strong relation between surface roughness and deposition angle. Researchers extended the study to present a robust model having capability to predict roughness an entire part surface as it previously differs on the horizontal, vertical, top and lower surface. Back propagation algorithmbased artificial neural network was used to evaluate the surface roughness taking five input parameters and this model was found highly efficient as compared to the previous one after validation studies. Some authors [12] used a method for data handling and differential evolution to develop a model for prediction of tensile strength using orientation angle, air gap, raster angle, and raster width as input parameters. Non-uniform rational B-spline model was used to calculate the local surface roughness of FDM parts based on cusp's geometric models. Based on polynomial interpolation of the massive data, the predicted and experimental values were found in agreement. The average surface roughness model was developed in terms of layer thickness and orientation and angle [13]. Raju et al. [14] implemented a hybrid particle swarm (PSO) and bacterial foraging optimization evolutionary (BFO) algorithm. Initially, the 18 parts were manufactured using the Taguchi design of experiments technique, and the relationship between roughness and output parameters was formed through linear multiple regression. Afterwards, optimization of process parameters was implemented to identify
$0.07 \mathrm{~mm}$ layer thickness and $60^{\circ}$ orientation angle as best parametric levels to achieve maximum hardness, flexural modulus, tensile strength on FDM part surface.

Although commercial FDM printers fabricate components with a tolerance of $\pm 0.1 \mathrm{~mm}$, but recent optimization studies have reported an improvement of dimensional accuracy of complex designs. Pennington et al. [15] reported the minimum dimensional variability of $0.05 \mathrm{~mm}$ in the length of benchmark parts.

Conventionally, techniques such as analysis of variance, taguchi, response surface methodology, and regression methods were employed for optimization of manufacturing process parameters. Presently, advanced optimization techniques such as artificial neural networks, genetic algorithms, neuro-fuzzy techniques, and different algorithms are also used by researchers to achieve desired output characteristics. Most of the aforementioned studies are focused on the improvement of surface finish and mechanical properties using optimization algorithms. However, the impact of advanced optimization algorithms on dimensional accuracy, warp deformation, and manufacturing time has not been extensively explored. Moreover, the Learning enthusiasm based Teaching-learning based algorithm (TLBO) has been successfully implemented by researchers in other areas [16] but not been implemented on problems related to FDM technology.

The present study aims to optimize the process parameters of FDM technology using learning enthusiasm based TLBO. The study would utilize advanced TLBO optimization tool to minimize dimensional variability, warp deformation, and manufacturing time. The study would validate the efficacy of advanced TLBO algorithm for manufacturing issues along with reduce rejection rate and manufacturing time during mass production of FDM products.

The next section explains the fundamentals of TLBO along with advanced features added to learning enthusiasm based TLBO. Section 3 elaborates the detailed methodology, materials used, and procedure adopted for the selection of input and output parameters. In Sect. 4, simulation results and comparative analysis of five conventional algorithms with learning enthusiasm based TLBO are presented. In Sect. 5, the findings of confirmatory experiments are discussed which are performed to compare the experimental and predicted results.

\section{Learning enthusiasm based TLBO (LebTLBO)}

Rao et al. [17] proposed a metaheuristic algorithm called TLBO influenced by teaching and learning behaviour at standard conditions. The motivation behind this system is the simulation of methodology and process adopted 
during classroom teaching and learning method. TLBO uses two innovative operators, termed as teacher and learning phase to look for good solutions [18]. TLBO's greatest advantage is its engaging characters, such as easy to execute and fast convergence. It has received strong attention and has been expanded to deal with constrained objective, large-scale, and dynamic optimization problems [19]. Several engineering problems, such as neural network training, power grid dispatch, and output scheduling have been discussed using TLBO variants [20-22].

An enhanced version of the basic TLBO is Learning enthusiasm based TLBO (LebTLBO). LebTLBO increases the search ability for an optimal solution. LebTLBO introduced two new modules or stages and the first stage comprise of learning enthusiasm based teacher part while the next stage is fundamentally a learner part. The first stage is employed to increase the search capacity, in order to improve the quality of poor learners using poor student tutoring phase. Every learner has the same ability to acquire information from others in basic TLBO [23]. However, LebTLBO is guided by a process of real-world learning excitement, where each learner has different abilities and excitement or eagerness to learn. This process of learning excitement motivates LebTLBO.

The algorithm in its initial stage consists of a population of NP learners (where the total population is denoted by $x)$, initialized as Eq. (1):

$x_{i}^{j}=x_{\text {min }}^{j}+a_{b} *\left(x_{\text {max }}^{j}-x_{\text {min }}^{j}\right)$

where $i \in\{1,2, \ldots . N P\}, j \in\{1,2, \ldots . . D\}, x_{i, j}$ is the ithsolution in the $j$ th dimension; $a_{b}$ is random number between $[0,1] ; x$ ${ }_{\text {min' }} x_{\max }$ are lower bound and upper bound respectively. Here, $D$ is dimension of the problem under test conditions.

After initializing the population of learners, each learner's fitness is evaluated. The fitness calculated from given solutions is taken as final results or grades. The learner with the best fitness is identified as the teacher $x_{\text {teacher }}$. LebTLBO algorithm is, basically a combination of three distinct phases out of which Learning enthusiasm based teacher phase is the initial and most critical stage. Afterwards, the implementation of Learning enthusiasm based learner phase is done and assessed. In the last phase, the poor student tutoring stage is executed to calculate the worst performance.

\subsection{Learning enthusiasm based teacher phase}

In this phase, the students with good assessments have more learning excitement and exhibit a large probability of understanding the instructor's information, while students with poor assessments exhibit less learning motivation and have little probability of understanding the educator's information.

In this phase, the entire group of learners are sequenced from best to worst depending upon their grades. For a minimization problem, Eq. (2) is taken:

$f\left(x_{1}\right) \leq f\left(x_{2}\right) \leq \ldots . \leq f\left(x_{N P}\right)$

Afterwards, the learning enthusiasm data of students are calculated using formula (3):

$L E_{i}=L E_{\min }+\left(L E_{\max }-L E_{\min }\right) \frac{N P-i}{N P}$

$i=1,2, \ldots, N P$.

In this equation, $L E_{\min }$ and $L E_{\max }$ are the minimum and maximum learning enthusiasm of learner respectively. As illustrated in the learning enthusiasm curve (see Fig. 3), the worst learner shows the lowest learning enthusiasm while the best learner displays the highest learning enthusiasm.

Depending on the value of learning enthusiasm $L E_{i}$, each student is graded into the category of benefit or learning from the teacher or not learning from the teacher in the wake of characterising the learning enthusiasm. A random number $r_{i} \in[0,1]$ is generated for each student $x_{i}$, in the event that $r_{i} \leq L E_{i}$, at that point student $x_{i}$ will benefit from the teacher; otherwise a student $x_{i}$ will usually ignore the teacher's learning. If student $x_{i}$ is able to obtain the instructor's knowledge, the position is refreshed using the methodology as shown in Fig. 4 and the accompanying condition (4) which is the hybridization of TLBO and DE [24].

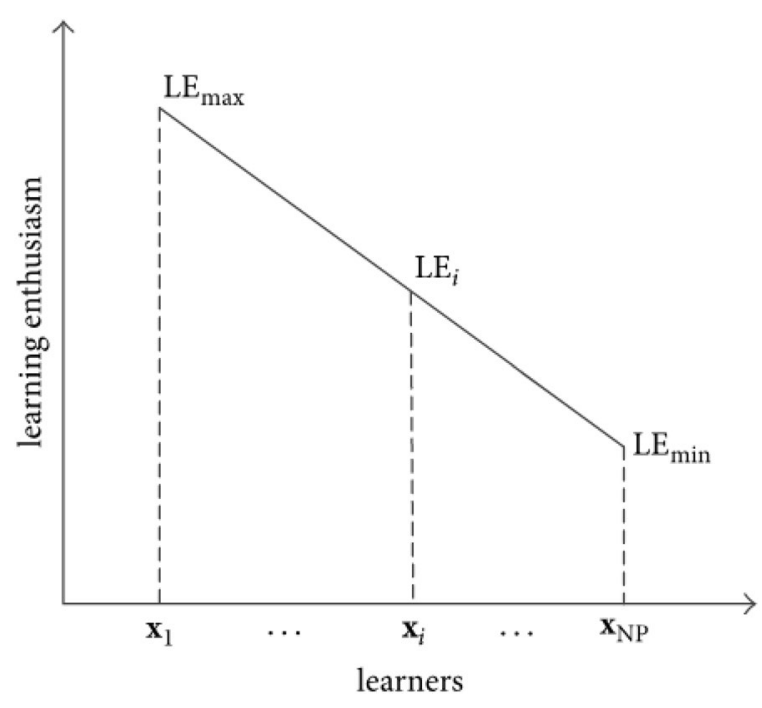

Fig. 3 Learning enthusiasm curve [24] 
Fig. 4 Diversity enhanced teaching strategy [24]

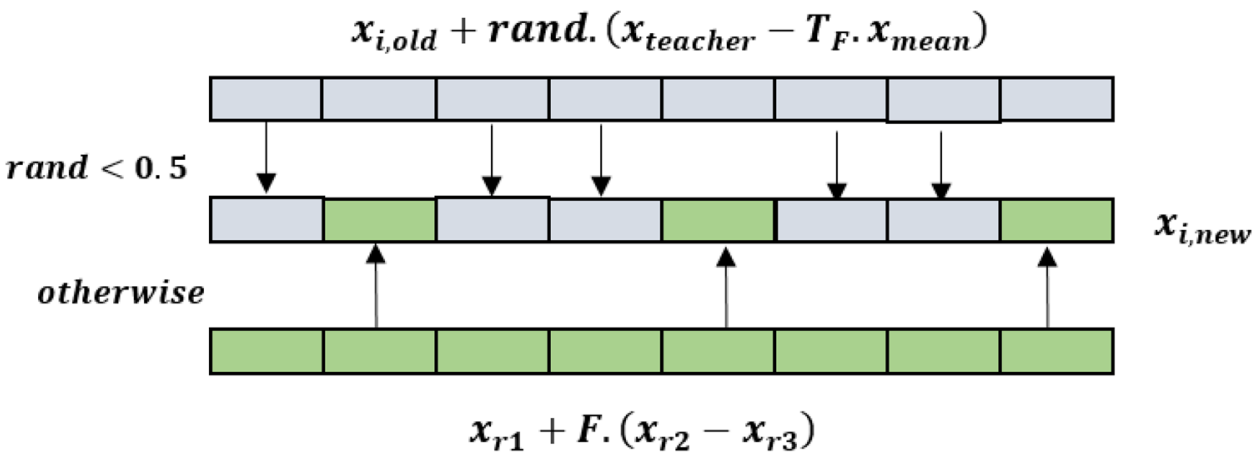

$x_{i, \text { new }}^{d}=\left\{\begin{array}{lr}x_{i, 0 \text { ld }}^{d}+\operatorname{rand}_{2} \cdot\left(x_{\text {teacher }}^{d}-T_{F} \cdot x_{\text {mean }}^{d}\right) & \text { if rand } d_{1}<0.5 \\ x_{r_{1}}^{d}+F \cdot\left(x_{r_{2}}^{d}-x_{r_{3}}^{d}\right) & \text { otherwise }\end{array}\right.$

where $r_{1}, r_{2}$ and $r_{3}\left(r_{1} \neq r_{2} \neq r_{3} \neq i\right)$ are randomly chosen integers given as $\{1,2, \ldots, N P\} ; d \in\{1,2, \ldots, D\}$; rand $_{1}$ and rand $_{2}$ are randomly generated numbers which are uniformly dispersed within $[0,1]$; and $F$ is a scale factor which lies within $[0,1]$.

\subsection{Learning enthusiasm based learner phase}

Similar to the teaching strategy discussed earlier, it entails greater learning excitement for learners with good grades and the place of those learners in a high-probability area for understanding from others and vice versa. All learners are graded in the range from best to worst based on the performance of grades.

For learner $x_{i}$, a random number is generated between $r_{i} \in[0,1]$, if $r_{i} \leq L E_{i}$, then learner $x_{i}$ will learn from the other learner; otherwise the learner's understanding will be ignored by the learner $x_{i}$. If learner $x_{i}$ will receive the teacher's expertise, his position will be modified based on a diversity-enhanced teaching approach.

$x_{i, \text { new }}= \begin{cases}x_{i, \text { old }}+\text { rand } .\left(x_{i}-x_{j}\right), & \text { if } f\left(x_{i}\right) \geq f\left(x_{j}\right) \\ x_{i, \text { old }}+\text { rand } .\left(x_{j}-x_{i}\right), & \text { if } f\left(x_{i}\right)<f\left(x_{j}\right)\end{cases}$

where rand is a random vector uniformly dispersed between $[0,1], f\left(X_{i}\right)$ is the fitness function, $X_{i, \text { old }}$ is the previous position of the ith learner as by Eq. (5). If $x_{i, \text { new }}$ is better than $x_{i, \text { old }}$ then $x_{i, \text { new }}$ is accepted, otherwise $x_{i, \text { old }}$ is unchanged.

\subsection{Poor student tutoring phase}

This phase cannot be found while using conventional TLBO, the idea behind this stage is to enhance the performance of weak students. In this point, all students are sorted again in the range from best to worst based on their grades.
The students lie in the bottom $10 \%$ grades are considered as poor learners. From any poor student xi whose rank is in the top 50 percent, this stage will pick learner $x_{T}$ randomly and the learning is based on the following Eq. (6).

$x_{i, \text { new }}^{d}=x_{i, \text { old }}^{d}+\operatorname{rand} .\left(x_{T}^{d}-x_{i, \text { old }}^{d}\right)$

If $x_{i, \text { new }}$ is better than $x_{i, \text { old }}, x_{i, \text { new }}$ is approved, $x_{i, \text { old }}$ would otherwise be unchanged. Students with poor grades are less likely to update their position in the good student group, while students with good grades are relatively more likely to update their position. The poor student tutoring process plays a significant role in raising the poor student's grades to a good student. This approach is applicable to the real-time teaching-learning process in which, relative to other good students, bad students often need tutorials for their progress. Figure 5 displays the flowchart for LebTLBO. The methodology has been implemented in present study where process parameters of FDM technology are optimized using LebTLBO and results are compared with other optimization algorithms. The case study has been conducted to identify optimum parametric settings to achieve minimum dimensional variability, deformation and manufacturing time. The detailed methodology has been discussed in next section.

\section{Methodology and selection of FDM parameters}

In the present study, four input parameters of FDM have been selected based on an optimization study conducted by Peng et al. [25]; these parameters are line width compensation, extrusion speed, filling speed and layer thickness. Limited research has been found where the combined impact of these four parameters has been studied on dimensional accuracy, warp deformation, and manufacturing time. Moreover, LebTLBO has not been implemented to solve dimensional accuracy issues of FDM technology which increases the need to execute this case 


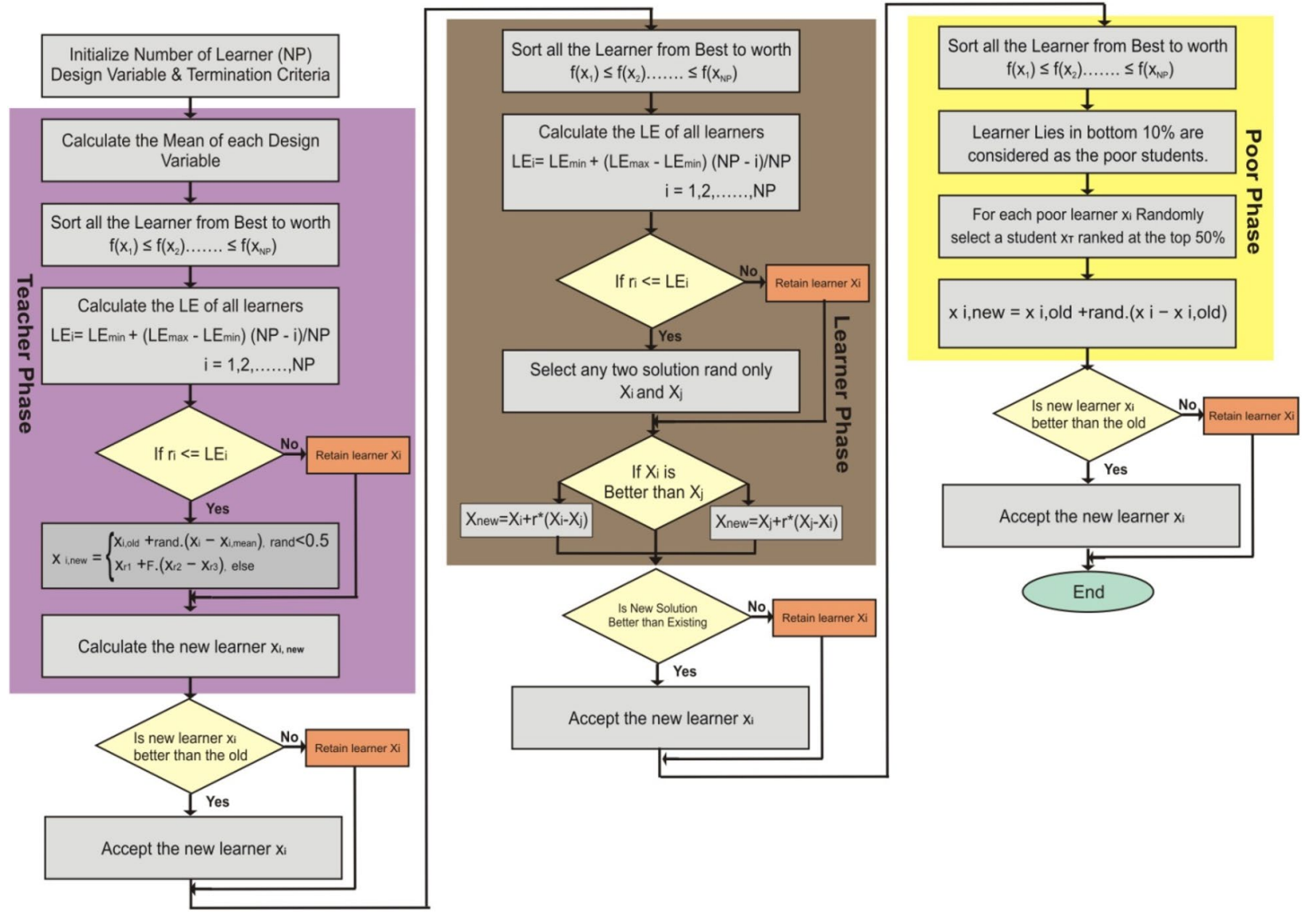

Fig. 5 Flowchart of implementation of LebTLBO for present study

study. The secondary data has been used to generate equations from the optimization study conducted by Peng et al. [25]. The thermoplastic material considered for the present case study is Acrylonitrile Butadiene Styrene (ABS).

During fabrication of polymer parts in FDM, the material is extruded in the form of semi-molten beads and after cooling minute shrinkage in a material is experienced. This leads to a significant difference between theoretical and actual contour parts as compensation due to shrinkage is provided which is called line width compensation $\left(x_{1}\right)$. Extrusion speed $\left(x_{2}\right)$ is the rate at which plastic filament is extruded by extrusion head during layer deposition. On contrary, filling speed $\left(x_{3}\right)$ is the movement of nozzle per unit time during part fabrication under FDM extruder head. Another variable parameter in the present case study is layer thickness $\left(x_{4}\right)$ which is used to develop an empirical relationship between input parameters and response parameters. In case the filling speed is lesser than the extrusion speed, the material expands and sticks to outer conical surface of nozzle leading to the part failure. Moreover, lower filling speed results in reduced manufacturing efficiency due and higher heat exposure in nozzle while higher filling speed leads to dangerous vibrations in machine. On the other hand, if extrusion speed is lesser than filling speed, material thinning is experienced due to dragging which also distorts part accuracy. Although the head temperature has significant impact on dimensional accuracy, but increase in temperature beyond $230^{\circ} \mathrm{C}$ resulted in excessive flowability of filament which widens after deposition, thus deteriorating the accuracy. Thus, head temperature has been maintained at constant value of $230^{\circ} \mathrm{C}$ which is optimum as per previous studies. On the other hand, it is obligatory to identify the optimum combination of filling and extrusion speed for a safe and efficient fabrication process. Moreover, layer thickness significantly impacts manufacturing time while line width compensation has a significant impact on dimensional accuracy and warp deformation.

The three response parameters i.e. dimensional accuracy, warp deformation, and manufacturing time have been selected. Dimensional accuracy must be enhanced to enable usability of FDM components for rapid tooling, biomedical engineering, and direct use applications. The variability of dimensions of few microns in medical implants may lead to postoperative complications; thus, it is a critical response parameter that must be 
investigated. Warp deformation is significantly affected by extrusion temperature, but other factors such as printing speed, layer thickness, and width compensation also have a positive influence. Excessive warping directly deteriorates the accuracy, aesthetic value and usability of FDM parts. Thus, it is obligatory to optimize input parameters to minimize warp deformation. On the other hand, manufacturing time must be minimized to increase the production rate and lower manufacturing cost. Moreover, the challenge of an increasing demand for customized products can be converted into opportunity by minimizing manufacturing time. These three response parameters are converted to a single response (y) utilizing MATLAB software. In the present study, the objective function is expressed by Eq. (7).

$$
\begin{aligned}
y= & 806-33763.1 x_{1}+225.30 x_{2}+14.81 x_{3}-2759.88 x_{4} \\
& +67560.93 x_{1}^{2}-3.57 x_{2}^{2}+0.17 x_{3}^{2}+10607.87 x_{4}^{2} \\
& +17.16 x_{1} x_{2}+172.22 x_{1} x_{3}-1.86 x_{2} x_{3}+5.57 x_{2} x_{4}-61.53 x_{3} x_{4}
\end{aligned}
$$

The parameter bounds are expressed by Eqs. (8-11).

$0.17 \mathrm{~mm} \leq x_{1} \leq 0.25 \mathrm{~mm}$

$20 \mathrm{~mm} / \mathrm{s} \leq x_{2} \leq 30 \mathrm{~mm} / \mathrm{s}$

$20 \mathrm{~mm} / \mathrm{s} \leq x_{3} \leq 40 \mathrm{~mm} / \mathrm{s}$

$0.15 \mathrm{~mm} \leq x_{4} \leq 0.30 \mathrm{~mm}$

The method used for the conversion of three mathematical equations into single equation is weighted sum model. Here, $\mathrm{Wj}$ is assigned to criterion $\mathrm{Cj}$ as a relative weight and aij is assigned to alternative $A i$ as a performance value during computation. Hence, when each criterion is considered at same instant for the importance of alternative $\mathrm{A} i$, the weighted sum model is given by Eq. (12):
$A_{i}^{W S M-S c o r e}=\sum_{j=1}^{n} w_{j} a_{i j}$

$i=1,2,3,4 \ldots \ldots n$

Here, equal weightage is given to all the three response parameters as each feature is paramount in case of mass production of FDM components.

\section{Simulation results}

LebTLBO has been implemented in the present case study to solve the optimization problem in the FDM process. Because heuristic algorithms are stochastic optimization methods, they must be run at least more than 10 times to produce useful statistical results. For this reason, each simulation is carried out 30 times, with a population size of 30 and a maximum number of iterations of 500 . TLBO, Cukoo search (CS), Novel Bat Algorithm (NBA), Grey Wolf Optimizer (GWA) and Dragonfly Algorithm (DA) algorithms are selected for comparison and evaluate the performance of LebTLBO algorithm [26-29]. These specific optimization tools and algorithms are used for comparison as these are previously used for the manufacturing domain and quite popular among production engineers. But, LebTLBO was not previously used for optimization of FDM and other Additive manufacturing parameters. Thus, it was imperative to compare these popular algorithms with LebTLBO. The parametric settings of given algorithm used for comparison is shown in Table 1. Note that for each of the algorithms, we used 30 search agents and 500 iterations.

Tables 2, 3 include the optimum and worst solutions respectively obtained by simulated algorithms. From these findings, it is evident that the fitness values of the LebTLBO are marginally better than competitive algorithms. The convergence rate of the LebTLBO is also higher than the other algorithms, as shown in Fig. 6 . The performance

Table 1 Parameter settings

\begin{tabular}{ll}
\hline Algorithm & Parameters \\
\hline Teaching learning based optimization (TLBO) & $N P=30 ; D=4 ; G_{\text {max }}=500$ \\
Cuckoo search (CS) & $N P=30 ; D=4 ; G_{\text {max }}=500 ;$ Probability $\left(p_{a}\right)=0.25$ \\
Novel bat algorithm (NBA) & $N P=30 ; D=4 ; G_{\max }=500 ; A=0.5 ; r=0.5 ; a=\gamma=0.9 ; f_{\min }=0 ; f_{\max }=1.5$ \\
Grey wolf optimization (GWO) & $N P=30 ; D=4 ; G_{\text {max }}=500 ; a=[2-0]$ \\
Dragonfly algorithm (DA) & $N P=30 ; D=4 ; G_{\text {max }}=500 ; w=[0.4-0.9], s=0.1, a=0.1, c=0.7, f=1, e=1$ \\
Learning enthusiasm-teaching learning based optimization (LebT- & $N P=30 ; D=4 ; G_{\max }=500$ \\
\hline LBO) & \\
\hline
\end{tabular}

$N P$ number of population, $D$ dimension of population

Gmax is number of iteration 
Table 2 Results of different optimization tools for population size 30

\begin{tabular}{lllllll}
\hline $\begin{array}{l}\text { Process } \\
\text { Parameters }\end{array}$ & Units & TLBO & CS & NBA & GWO & DA \\
\hline$x_{1}$ & $\mathrm{~mm}$ & 0.25 & 0.25 & 0.25 & 0.25 & 0.25 \\
$x_{2}$ & $\mathrm{~mm} / \mathrm{s}$ & 21.8523 & 21.8523 & 21.8523 & 21.8523 & 21.8523 \\
$x_{3}$ & $\mathrm{~mm} / \mathrm{s}$ & 40 & 40 & 40 & 40 & 40 \\
$x_{4}$ & $\mathrm{~mm}$ & 0.15 & 0.15 & 0.15 & 0.15 & 0.15 \\
$y$ & - & 334.657069065128 & 334.657069065128 & 334.657069065128 & 334.657069045030 & 334.657069065127 \\
\hline
\end{tabular}

Table 3 Values of worst solutions from different optimization tools for population size 30

\begin{tabular}{|c|c|c|c|c|c|c|c|}
\hline $\begin{array}{l}\text { Process } \\
\text { Parameters }\end{array}$ & Units & TLBO & $\mathrm{CS}$ & NBA & GWO & DA & LebTLBO \\
\hline$x_{1}$ & $\mathrm{~mm}$ & 0.17 & 0.25 & 0.17 & 0.17 & 0.25 & 0.25 \\
\hline$x_{2}$ & $\mathrm{~mm} / \mathrm{s}$ & 26.988 & 21.8523 & 26.9879 & 26.9883 & 21.8523 & 21.8523 \\
\hline$x_{3}$ & $\mathrm{~mm} / \mathrm{s}$ & 20 & 40 & 20 & 20 & 40 & 40 \\
\hline$x_{4}$ & $\mathrm{~mm}$ & 0.30 & 0.15 & 0.30 & 0.30 & 0.15 & 0.15 \\
\hline$y$ & - & 326.148167142859 & 334.657069065128 & 326.148167142854 & 326.148137531163 & 334.657069065127 & 334.657069065128 \\
\hline
\end{tabular}

Fig. 6 Convergence curves for LebTLBO for a population size of 30

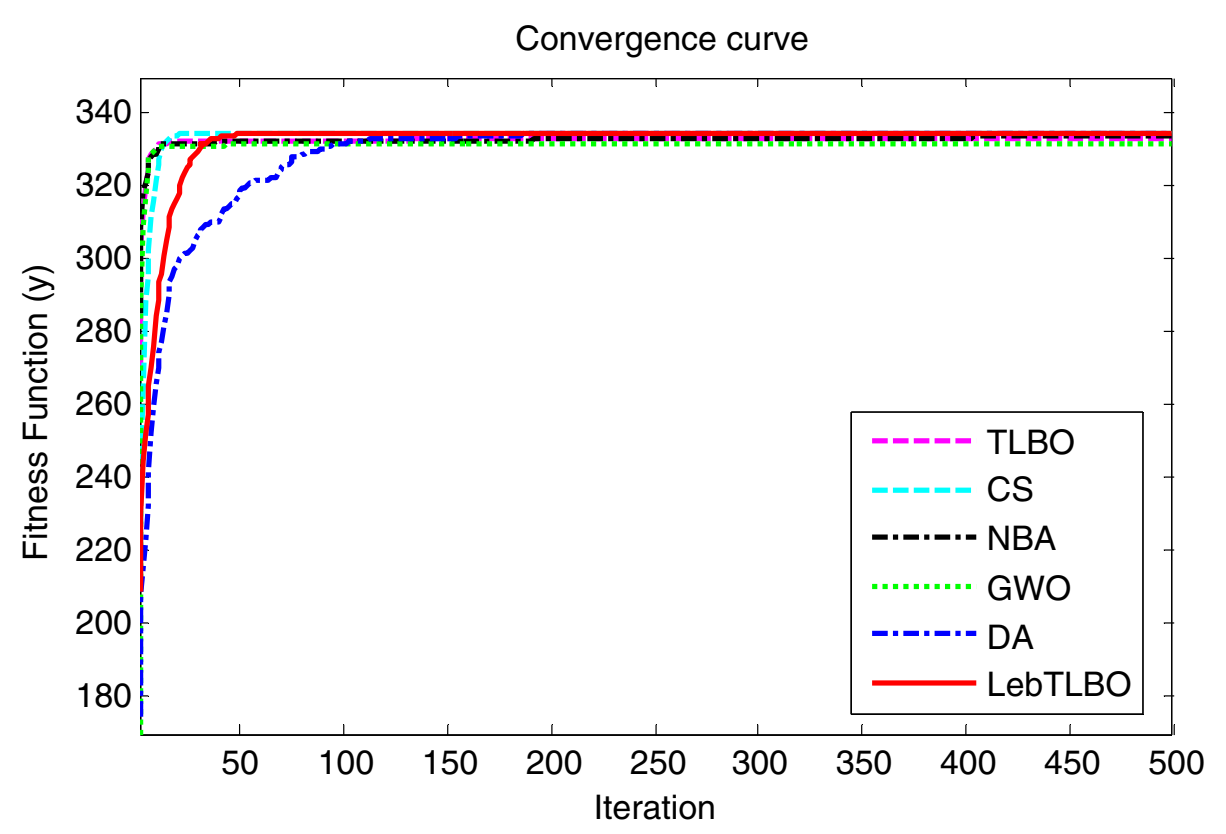

of simulated algorithms for FDM is given in Table 4, which shows that the mean, median, and standard deviation values of LebTLBO are much better compared to competitive algorithms that demonstrate the proposed algorithm's enhanced exploration and exploitation capabilities.

Figure 7 also draws the box-plot of competitive algorithms for the problem under study. The box-plots here are used to measure algorithm efficiency in terms of fitness values. It can be shown that the LebTLBO algorithm is cost-effective in fitness value as their median of fitness values is higher and standard deviation is least.
Therefore, its overall performance is found to be consistent and better than other algorithms for optimization.

It is quite evident from Table 4 results that learning enthusiasm based algorithm has given best output as compared to other tools. The response would be maximum if $0.25 \mathrm{~mm}$ line width compensation is used with layer thickness of $0.15 \mathrm{~mm}$ and $21.85 \mathrm{~mm} / \mathrm{s}$ extrusion speed and $40 \mathrm{~mm} / \mathrm{s}$ filling speed. The maximum output of $y=334.657069065128$ has been given at optimum at of parameters which is normally greater than other optimization tools. 
Table 4 Output of simulated optimization tools over 30 independent runs

\begin{tabular}{llllll}
\hline Algorithm & Best & Worst & Mean & Median & Std. dev \\
\hline TLBO & 334.657069065128 & 326.148167142859 & 333.156734827457 & 334.657069065127 & 3.21931536685901 \\
CS & 334.657069065128 & 334.657069065127 & 334.657069065127 & 334.657069065127 & $1.35998849951065 \mathrm{e}-13$ \\
NBA & 334.657069065128 & 326.148167142854 & 333.522192414360 & 334.657069065127 & 2.94177639853746 \\
GWO & 334.657069045030 & $\mathbf{3 2 6 . 1 4 8 1 3 7 5 3 1 1 6 3}$ & 331.506679256138 & 334.657048024297 & 4.15020799011596 \\
DA & 334.657069065127 & 334.657069065127 & 334.657069065127 & 334.657069065127 & $1.35998849951065 \mathrm{e}-13$ \\
LebTLBO & $\mathbf{3 3 4 . 6 5 7 0 6 9 0 6 5 1 2 8}$ & 334.657069065128 & $\mathbf{3 3 4 . 6 5 7 0 6 9 0 6 5 1 2 8}$ & $\mathbf{3 3 4 . 6 5 7 0 6 9 0 6 5 1 2 8}$ & $\mathbf{1 . 0 1 2 4 5 3 5 4 5 3 5 6 1 8 e - 1 3}$ \\
\hline
\end{tabular}

Best values are given in bold

Fig. 7 Box plot for FDM problem under study

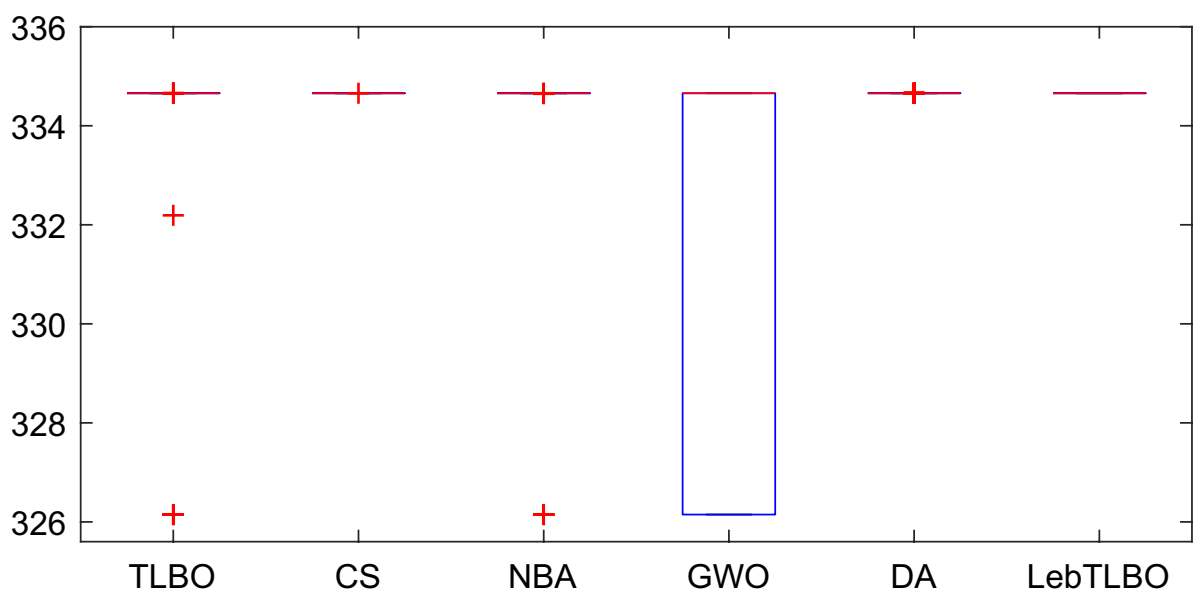

Moreover, the tool used in the present study gives same output both in worst and optimum solutions which proves the effectiveness of tools while solving problems of Additive manufacturing. The tool has been highly recommended for further use in optimization studies related to surface finish, mechanical strength, dimensional accuracy, and manufacturing time and cost of FDM technique. This would help to rapidly find best solutions for numerous problems experienced during using these technologies for practical applications. Moreover, the minimum risk is required while developing biomedical implants and aerospace parts and thus, this advanced algorithm can be successfully used for optimization problems within minimum time and cost constraints.

\section{Confirmatory experiments}

The confirmatory experiments have been conducted to validate the simulation results; hence fabrication of samples was performed at variable input parameters. The rectangular-shaped samples $(10 \mathrm{~cm} \times 3 \mathrm{~cm} \times 0.5 \mathrm{~cm})$ were prepared using $A B S$ thermoplastic material through open-source FDM printer (Make: Prusa I3) as shown in Fig. 8. The required modifications were made in the FDM

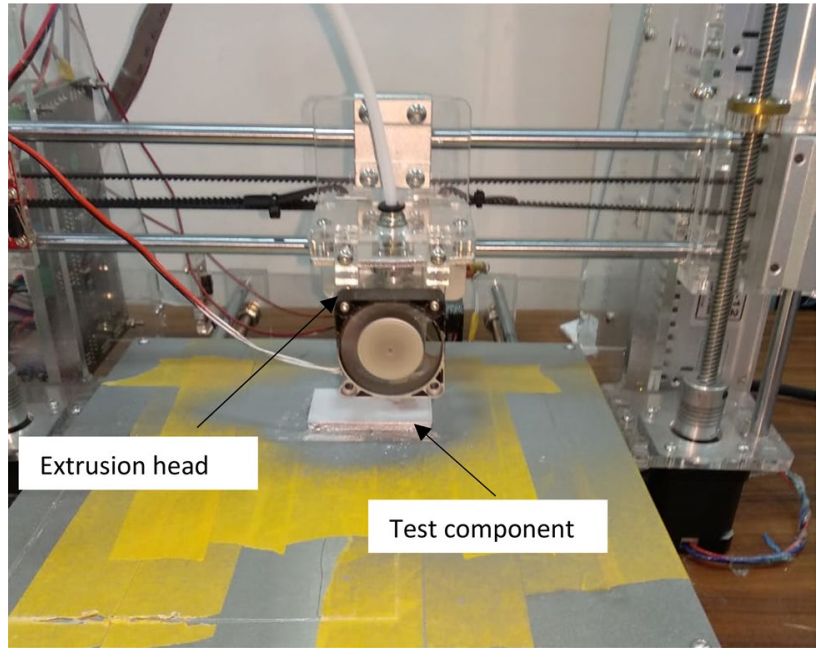

Fig. 8 FDM printer used for experimentation

printer assembly for operation at variable parameters. The fabrication of test components was performed at $0^{\circ}$ orientation angle, raster and contour width $0.4070 \mathrm{~mm}$, $0^{\circ} / 90^{\circ}$ raster angle, $80^{\circ} \mathrm{C}$ bed temperature and $310^{\circ} \mathrm{C}$ extrusion temperature. 
The percentage change in dimensional accuracy along with percentage warp deformation was measured using digital Vernier caliper (Make: Mitutoyo) using following formulas:

Percentage Warp Deformation $=$ Centre height

- Corner height/Centre height $x 100$

Percentage change in Dimensional Accuracy

$=$ Initial width - final width/initial width $\times 100$

The variable parameters for confirmatory experiments are width compensation, extrusion speed, filling speed and layer thickness. The range of these parameters is selected as given in Eqs. 8-11. The rectangular parts are prepared at variable process parameters using full factorial experimental design. The measurements were repeated thrice and the average was considered as final value; afterwards, percentage change was calculated using Eqs. (13, 14). Table 5 displays different levels of variable parameters considered for present experimentation.

The variation in warp deformation and dimensional accuracy at different line width and layer thickness

Table 5 Levels of variable process parameters

\begin{tabular}{lllll}
\hline S. No & $\begin{array}{l}\text { Line Width Com- } \\
\text { pensation }(\mathrm{mm})\end{array}$ & $\begin{array}{l}\text { Extrusion } \\
\text { Speed } \\
(\mathrm{mm} / \mathrm{s})\end{array}$ & $\begin{array}{l}\text { Filling } \\
\text { Speed } \\
(\mathrm{mm} / \mathrm{s})\end{array}$ & $\begin{array}{l}\text { Layer Thick- } \\
\text { ness }(\mathrm{mm})\end{array}$ \\
\hline 1 & 0.17 & 20 & 20 & 0.15 \\
2 & 0.19 & 22 & 25 & 0.18 \\
3 & 0.21 & 24 & 30 & 0.21 \\
4 & 0.23 & 26 & 35 & 0.24 \\
5 & 0.25 & 28 & 40 & 0.27 \\
\hline
\end{tabular}

settings have been plotted in Fig. 9. It can be observed that percentage error in dimensions and warp deformation occurred near predicted values of line width and layer thickness. Simulation results derived from LebTLBO were compared with actual experimental data and minimum error was found in case of line width compensation $(0.67 \%)$ and layer thickness (3.42\%).

Furthermore, experiments conducted at variable extrusion speed and filling density to evaluate their impact on dimensional accuracy and warp deformation as shown in Fig. 10. In this case, minimum values of dimensional accuracy and warp deformation occurred at extrusion speed and filling speed which is very close to predicted values with variation of $1.28 \%$ and $2.76 \%$ respectively.

Table 6 summarizes the findings of experimentation at optimum parametric settings. The experimental data is compared with predicted data and minimal error (7.42\%) was recorded in case of warp deformation estimation. However, $11.74 \%$ error was found between predicted and experimental results in case of dimensional accuracy which is higher than warp deformation estimation but lesser than other experimental studies. This indicted that LebTLBO is an efficient tool to predict the optimum parameters and solve complex manufacturing issues.

\section{Conclusions}

In this research work, efforts have been made to evaluate the efficiency of advanced learning enthusiasm based teaching-learning algorithm for optimization of FDM parameters. In comparison to conventional optimization algorithms, learning enthusiasm based algorithm is most effective for identifying the best solution, mean,

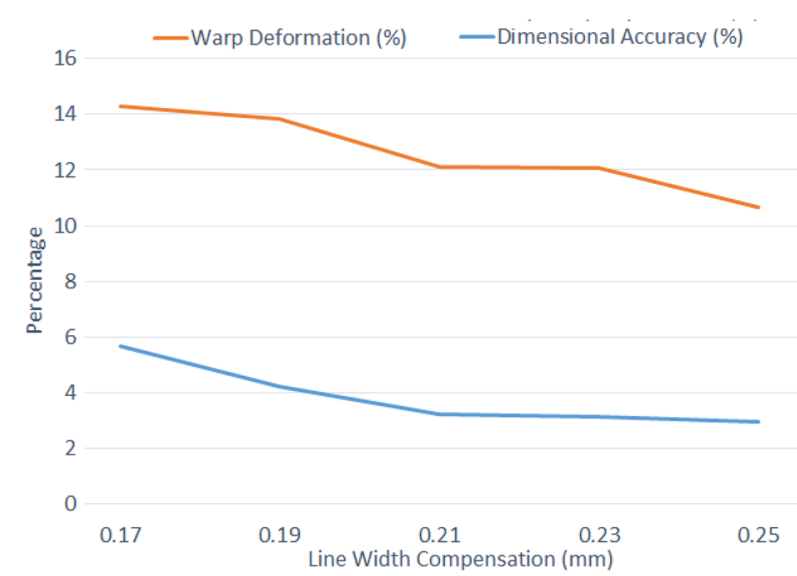

(a)

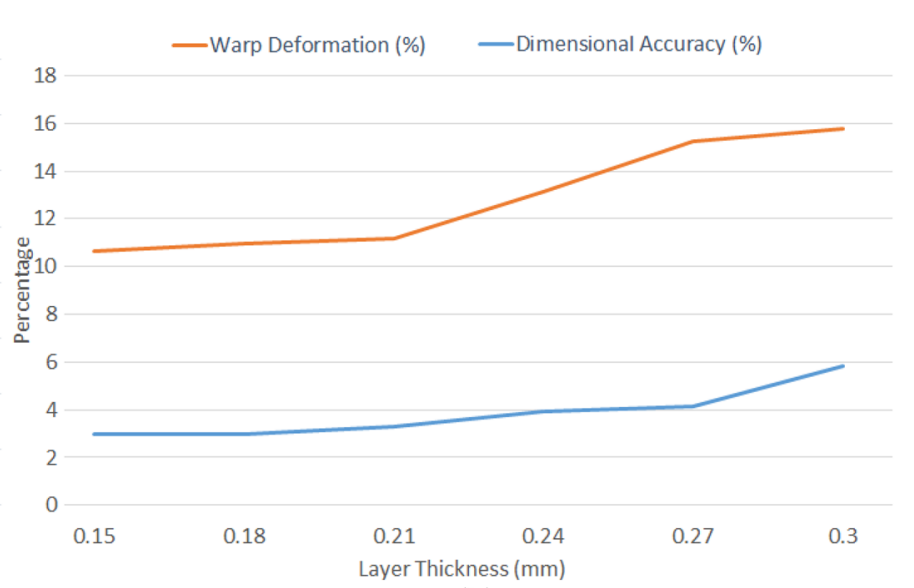

(b)

Fig. 9 Measured dimensional accuracy and deformation at variable $\mathbf{a}$ Line width compensation and $\mathbf{b}$ layer thickness 


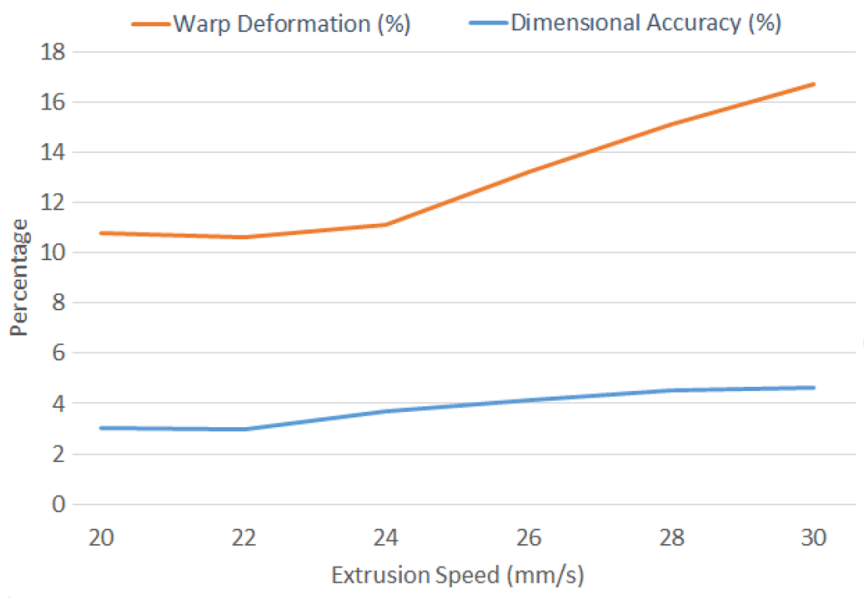

(a)

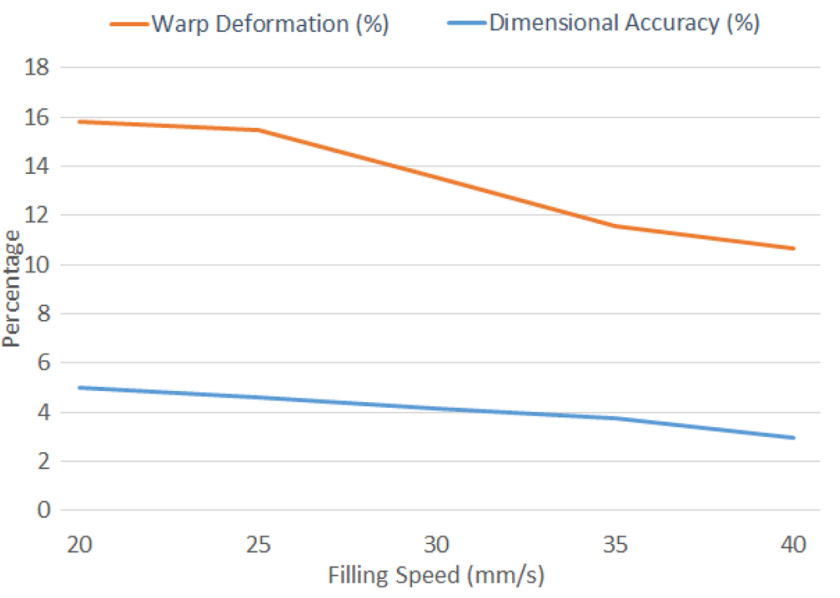

(b)

Fig. 10 Measured dimensional accuracy and deformation at variable a Extrusion Speed and b Filling Speed

Table 6 Summary of optimum parameters and comparison of predicted verses modelled data

\begin{tabular}{|c|c|c|c|c|c|c|c|c|c|}
\hline \multirow{2}{*}{$\begin{array}{l}\text { Line width } \\
\text { compensa- } \\
\text { tion (mm) }\end{array}$} & \multirow{2}{*}{$\begin{array}{l}\text { Extrusion } \\
\text { Speed } \\
(\mathrm{mm} / \mathrm{s})\end{array}$} & \multirow[t]{2}{*}{$\begin{array}{l}\text { Filling speed } \\
(\mathrm{mm} / \mathrm{s})\end{array}$} & \multirow[t]{2}{*}{$\begin{array}{l}\text { Layer thick- } \\
\text { ness (mm) }\end{array}$} & \multicolumn{2}{|c|}{ Warp deformation (\%) } & \multirow[t]{2}{*}{$\begin{array}{l}\text { Percentage } \\
\text { error }\end{array}$} & \multicolumn{2}{|c|}{$\begin{array}{l}\text { Dimensional accuracy } \\
\text { (\%) }\end{array}$} & \multirow[t]{2}{*}{$\begin{array}{l}\text { Percentage } \\
\text { error }\end{array}$} \\
\hline & & & & $\begin{array}{l}\text { Experimen- } \\
\text { tal }\end{array}$ & Predicted & & $\begin{array}{l}\text { Experimen- } \\
\text { tal }\end{array}$ & Predicted & \\
\hline 0.25 & 22 & 40 & 0.15 & 10.64 & 9.85 & 7.42 & 2.98 & 2.63 & 11.74 \\
\hline
\end{tabular}

median and standard deviation. A case study has been successfully implemented to find the optimum combination of four input parameters of FDM to achieve maximum dimensional stability, minimum manufacturing time and warp deformation. As compared to other tools, the case study with proposed algorithm predicted maximum value of output ( $y=334.657069065128)$. The findings reveal that maximum response can be achieved at $0.25 \mathrm{~mm}$ line width, $21.85 \mathrm{~mm} / \mathrm{s}$ extrusion speed, $40 \mathrm{~mm}$ filling speed and $0.15 \mathrm{~mm}$ layer thickness. The study was limited to only four input variables but other parameters such as orientation angle, raster angle and density also influence the dimensional accuracy and warp deformation. These parameters must be included in forthcoming studies to find optimum solutions for dimensional stability of FDM parts. The scope for future exploration also includes enhancement of mechanical strength and surface finish using learning enthusiasm based teaching-learning algorithm. This algorithm must be utilized for the reduction of product development time and cost to enable mass production of FDM components for rapid tooling, medical implants, and end-use applications.

\section{Compliance with ethical standard}

Conflict of interest On behalf of all authors, the corresponding author states that there is no conflict of interest.

\section{References}

1. Rao RV, Rai DP (2016) Optimization of fused deposition modeling process using teaching-learning-based optimization algorithm. Eng Sci Technol Int J 19(1):587-603

2. Pandey PM, Thrimurthulu K, Reddy NV (2004) Optimal part deposition orientation in FDM by using a multicriteria genetic algorithm. Int J Prod Res 42(19):4069-4089

3. Gavali VC, Kubade PR, Kulkarni HB (2020) Mechanical and thermo-mechanical properties of carbon fiber reinforced thermoplastic composite fabricated using fused deposition modeling method. Mater Today Proc 22:1786-1795

4. Ahn D, Kim H, Lee S (2009) Surface roughness prediction using measured data and interpolation in layered manufacturing. J Mater Process Technol 209(2):664-671

5. Thrimurthulu KPPM, Pandey PM, Reddy NV (2004) Optimum part deposition orientation in fused deposition modeling. Int J Mach Tools Manuf 44(6):585-594

6. Harsha AS, Kumar CV (2020) Fused deposition modeling using 6-axis industrial robot. In: Shunmugam MS, Kanthababu M (eds) Advances in Additive Manufacturing and Joining. Springer, Singapore, pp 159-168. https://doi. org/10.1007/978-981-32-9433-2_13 
7. Hossain MS, Espalin D, Ramos J, Perez M, Wicker R (2014) Improved mechanical properties of fused deposition modelingmanufactured parts through build parameter modifications. J Manuf Sci Eng 136(6):061002

8. Boschetto A, Bottini L (2016) Design for manufacturing of surfaces to improve accuracy in fused deposition modeling. Robot Comput Integr Manuf 37:103-114

9. Dey A, Yodo N (2019) A systematic survey of FDM process parameter optimization and their influence on part characteristics. J Manuf Mater Process 3(3):64

10. Mahapatra SS, Sood AK (2012) Bayesian regularization-based Levenberg-Marquardt neural model combined with BFOA for improving surface finish of FDM processed part. Int J Adv Manuf Technol 60(9-12):1223-1235

11. Boschetto A, Giordano V, Veniali F (2013) Surface roughness prediction in fused deposition modelling by neural networks. Int J Adv Manuf Technol 67(9-12):2727-2742

12. Rayegani F, Onwubolu GC (2014) Fused deposition modelling (FDM) process parameter prediction and optimization using group method for data handling (GMDH) and differential evolution (DE). Int J Adv Manuf Technol 73(1-4):509-519

13. Barari A, Kishawy HA, Kaji F, Elbestawi MA (2017) On the surface quality of additive manufactured parts. Int J Adv Manuf Technol 89(5-8):1969-1974

14. Raju M, Gupta MK, Bhanot N, Sharma VS (2018) A hybrid PSOBFO evolutionary algorithm for optimization of fused deposition modelling process parameters. J Intell Manuf 30:2743-2758

15. Pennington RC, Hoekstra NL, Newcomer JL (2005) Significant factors in the dimensional accuracy of fused deposition modelling. Proc Inst Mech Eng Part E J Process Mech Eng 219(1):89-92

16. Natarajan E, Kaviarasan V, Lim WH, Tiang SS, Parasuraman S, Elango $S$ (2019) Non-dominated sorting modified teachinglearning-based optimization for multi-objective machining of polytetrafluoroethylene (PTFE). J Intell Manuf 31:911-935

17. Rao RV, Savsani VJ, Vakharia DP (2012) Teaching-learning-based optimization: an optimization method for continuous non-linear large scale problems. Inf Sci 183(1):1-15

18. Patel VK, Savsani VJ (2016) A multi-objective improved teaching-learning based optimization algorithm (MO-ITLBO). Inf Sci 357:182-200
19. Chen X, Mei C, Xu B, Yu K, Huang X (2018) Quadratic interpolation based teaching-learning-based optimization for chemical dynamic system optimization. Knowl Based Syst 145:250-263

20. Chen D, Lu R, Zou F, Li S (2016) Teaching-learning-based optimization with variable-population scheme and its application for ANN and global optimization. Neurocomputing 173:1096-1111

21. Niknam T, Golestaneh F, Sadeghi MS (2012) $\theta$-Multiobjective teaching-learning-based optimization for dynamic economic emission dispatch. IEEE Syst J 6(2):341-352

22. Keesari HS, Rao RV (2014) Optimization of job shop scheduling problems using teaching-learning-based optimization algorithm. Opsearch 51(4):545-561

23. Chen X, Xu B, Yu K, Du W (2018) Teaching-learning-based optimization with learning enthusiasm mechanism and its application in chemical engineering. J Appl Math. https://doi. org/10.1155/2018/1806947

24. Storn R, Price K (1997) Differential evolution-a simple and efficient heuristic for global optimization over continuous spaces. J Global Optim 11(4):341-359

25. Peng A, Xiao X, Yue R (2014) Process parameter optimization for fused deposition modeling using response surface methodology combined with fuzzy inference system. The International Journal of Advanced Manufacturing Technology 73(1-4):87-100

26. Yang XS, Deb S (2009) Cuckoo search via Lévy flights. In 2009 World congress on nature $\&$ biologically inspired computing (NaBIC) (pp 210-214). IEEE

27. Meng X-B et al (2015) A novel bat algorithm with habitat selection and Doppler effect in echoes for optimization. Expert Syst Appl 42:6350-6364. https://doi.org/10.1016/j.eswa.2015.04.026

28. Mirjalili S, Mirjalili SM, Lewis A (2014) Grey wolf optimizer. Adv Eng Softw 69:46-61

29. Mirjalili S (2015) Dragonfly algorithm: a new meta-heuristic optimization technique for solving single-objective, discrete, and multi-objective problems. Neural Comput Appl. https:// doi.org/10.1007/s00521-015-1920-1

Publisher's Note Springer Nature remains neutral with regard to jurisdictional claims in published maps and institutional affiliations. 